\title{
Ecologia da comunidade de metazoários parasitos da anchova Pomatomus saltator (Linnaeus) (Osteichthyes, Pomatomidae) do litoral do estado do Rio de Janeiro, Brasil ${ }^{1}$
}

\author{
José Luis Luque ${ }^{2}$ \\ Nilton Domingos Chaves ${ }^{2}$
}

\begin{abstract}
Community ecology of metazoan parasites of bluefish Pomatomus saltator (Linnaeus) (Osteichthyes, Pomatomidae) from the littoral of State of Rio de Janeiro, Brazil. Fifty-five specimens of bluefish, Pomatomus saltator (Linnaeus, $1766)$, collected at Cabo Frio $\left(23^{\circ} \mathrm{S}, 42^{\circ} \mathrm{W}\right)$, State of Rio de Janeiro, Brazil, between March 1995 and November 1995, were necropsied to study their infracommunities of metazoan parasites. Sixteen species of metazoan parasites were collected. All fish were parasitized by one or more metazoan. The digeneans were the majority of the parasite specimens collected, with $44.2 \%$, followed by the helminth larval stages (cestodes, acanthocephalans, and nematodes) with $40.6 \%$. The Simpson index for all parasite species was 0.097 , indicating lack of dominance by any species in the parasite community. Microcotyle pomatomi Goto, 1899 showed the higher frequency of dominance and mean relative dominance. The parasite species of $P$. saltator showed the typical overdispersed pattern of distribution. The majority of parasite species showed positive correlation between the host's total body length and prevalence or parasite abundance. Two species, Brachyphallus parvus (Manter, 1947) and Phocanema sp. have differences in their prevalences and abundances in relation to sex of the hosts. The mean diversity in the infracommunities of $P$. saltator was $H^{\prime}=1.243 \pm 0.521$, with correlation with the host's total length and without difference between male and female fish. The components of the parasite community of $P$. saltator showed overall positive association. All pairs conformed by ectoparasites and adult endoparasites species showed significant positive association or covariation between their abundances. Five pairs of endoparasites larval species showed positive association or covariation, and two pairs, showed negative association and covariation. The parasite community of $P$. saltator was dominated by species with high prevalence values, that composed a high number of associations, thus, is considered closer to the interactive type.
\end{abstract}

KEY WORDS. Pomatomidae, Pomatomus saltator, parasitic ecology, community structure, marine fish, bluefish, Brazil

A anchova, Pomatomus saltator (Linnaeus, 1766) é uma espécie pelágica, de hábitos alimentares carnívoros e que possui ampla distribuição geográfica. Sendo freqüente no litoral do sudeste brasileiro, esta espécie é considerada de grande importância comercial (FIGUEIREDO \& MENEZES 1980).

1) Trabalho desenvolvido com auxilio do CNPq. e da FAPERJ.

2) Curso de Pós-graduação em Parasitologia Veterinária, Departamento de Parasitologia Animal, Universidade Federal Rural do Rio de Janeiro. Caixa Postal 74508, 23851-970 Seropédica, Rio de Janeiro, Brasil.

E-mail: jlluque@domain.com.br 
A nível mundial, $P$. saltator foi alvo de vários estudos relacionados com os seus parasitos, principalmente com enfoque taxonômico. ANDERSON (1970) confeccionou uma lista de parasitos conhecidos para este peixe. No Brasil, registros taxonômicos de parasitos de anchovas foram feitos em TRAVASSOS et al. (1969), KOHN \& BÜHRNHEIM (1971), KOHN et al. (1971, 1992), GOMES et al. (1972), AMATO (1983a,b), RÊGO et al. (1983) e WALLET \& KoHN (1987). Destes trabalhos, o mais abrangente foi o de RÊGO et al. (1983), que além da taxonomia, incluiu alguns aspectos quantitativos sobre os parasitos de $P$. saltator. Recentemente, PoULIN (1995) e ROHDE et al. (1995), usaram amostras de parasitos de anchovas provenientes de outras latitudes, para trabalhos comparativos de cunho ecológico.

A riqueza parasitária registrada pelos autores acima mencionados, foi um indicativo na escolha da anchova para análise da sua comunidade parasitária. Recentes publicações sobre as comunidades parasitárias de peixes marinhos da região Neotropical, demonstraram a necessidade de continuar acumulando evidências da presença ou ausência de processos interativos nestas, uma vez que ainda não foi possivel detectar padrões comuns à estrutura e à composição das comunidades parasitárias destes peixes (GEORGE-NASCIMENTO \& IRIARTE 1989; LUQUE \& OLIVA 1993; LUQUE 1994, 1996; LUQUe et al. 1996a,b; TAKEMOTO et al. 1996; KNOFF et al. 1997).

Neste trabalho é apresentada uma análise da comunidade parasitária de $P$. saltator, com o intuito de avaliar a dinâmica das infrapopulações parasitárias, examinando seus relacionamentos com alguns fatores bióticos como o comprimento total e o sexo dos hospedeiros, seus relacionamentos interespecíficos e a diversidade das respectivas infracomunidades parasitárias.

\section{MATERIAL E MÉTODOS}

Entre março e novembro de 1995, foram necropsiados 55 espécimes de $P$. saltator, coletados em Cabo Frio $\left(23^{\circ} \mathrm{S}, 42^{\circ} \mathrm{W}\right)$, litoral do estado do Rio de Janeiro, Brasil. Os peixes examinados mediram: 40,6 $\pm 5,8(32,0-52,0) \mathrm{cm}$ de comprimento total, e pesaram $639,1 \pm 223,7(329,0-1110,0) \mathrm{g}$. A determinação dos peixes foi feita de acordo com FigueIREDO \& MENEZES (1980).

A abordagem ecológica da comunidade parasitária de $P$. saltator foi feita a nível dos seus componentes e das suas infracomunidades (EsCH et al. 1990). Os componentes das infracomunidades parasitárias foram classificados, de acordo com BUSH \& HolmES (1986), em espécies centrais (presentes em mais de dois terços dos hospedeiros), espécies secundárias (presentes em um a dois terços dos hospedeiros) e espécies satélites (presentes em menos de um terço dos hospedeiros). O índice de Simpson $C$ foi calculado para determinar a concentração para dominância na comunidade parasitária. A concentração para dominância é assumida quando $C$ $\geq 0,25$ (STONE \& PENCE 1978). A dominância de cada componente das infracomunidades parasitárias foi determinada mediante o cálculo da freqüência de dominância e da dominância relativa (número de espécimes de uma espécie/número total de espécimes de todas as espécies de cada infracomunidade), seguindo a metodologia de RoHDE et al. (1995). O quociente entre a variância e a abundância parasitária 
média (índice de dispersão) foi calculado para cada espécie de parasito com o intuito de determinar seu padrão de distribuição. $O$ grau de agregação foi obtido através do cálculo do índice de Green (LUDWIG \& REYNOLDS 1988).

O coeficiente de correlação por postos de Spearman $r$ s foi usado para determinar possíveis correlações entre o comprimento total do hospedeiro e a intensidade de infecção/infestação. O coeficiente de correlação de Pearson $r$ foi usado para determinar a possivel correlação entre o comprimento total do hospedeiro e a prevalência de infecção/infestação parasitária, com prévia transformação angular dos dados de prevalência (ZAR 1996) e separação das amostras dos hospedeiros em quatro intervalos de classe de $10 \mathrm{~cm}$. A influência do sexo na abundância e na prevalência das infecções parasitárias foi testada pela aproximação normal $Z$ do teste $U$ de Mann-Whitney e pelo teste exato de Fisher $(F)$, respectivamente.

A diversidade parasitária de cada infracomunidade foi calculada através do índice de Shannon $\left(H^{\prime}\right)$, sendo determinada sua possível variação em relação ao sexo (teste $t$ ) e ao comprimento total (coeficiente de correlação por postos de Spearman $r s$ ). Foi calculado também, para cada infracomunidade o índice de uniformidade de Pielou $(J)$ (LUDWIG \& REYNOLDS 1988). O teste de proporção das variâncias (VR) foi computado para determinar uma possível associação total entre os componentes das infracomunidades parasitárias (SCHLUTER 1984). As possíveis associações interespecíficas entre pares de espécies co-ocorrentes foram determinadas através do qui-quadrado, usando a correção de Yates quando necessário. Possível covariação entre a abundância parasitária das espécies que formavam as associações foram analisadas com o coeficiente de correlação por postos de Spearman rs (LUDWIG \& REYNOLDS 1988).

A terminologia ecológica usada é a recomendada por BusH et al. (1997). A análise incluiu somente as espécies com prevalência maior que 10\% (ver BuSH et al. 1990). Todos os valores que correspondem à média de alguma variável são acompanhados do respectivo desvio padrão. O nível de significância estatística adotado foi $P \leq 0,05$.

\section{RESULTADOS}

\section{Componentes da comunidade parasitária}

Todos os peixes estavam parasitados por no mínimo uma espécie de metazoário. Um total de 2219 espécimes de parasitos pertencentes à 16 espécies foram coletados, com abundância média de 40,3 . Os digenéticos foram o táxon maioritário com $44,2 \%$ do total de metazoários coletados, parasitando $47(85,4 \%)$ hospedeiros, com abundância média de 20,8 . O conjunto dos estágios larvares de helmintos coletados de $P$. saltator constituíram $40,6 \%$ do total de metazoários coletados, parasitando $46(83,6 \%)$ dos hospedeiros, com abundância média de 17,6.

De acordo com a sua prevalência, duas espécies de parasitos (um digenético e um monogenético) foram consideradas espécies centrais; sete secundárias (dois digenéticos, um monogenético, dois eucestóides, um acantocéfalo e um copépode) e sete satélités (um digenético, um eucestóide, quatro nematóides e um copépode) (Tab. I). A comunidade parasitária não apresentou concentração para dominância 
$(C=0,097)$. Dentre os componentes das infracomunidades, Microcotyle pomatomi apresentou a maior frequêencia de dominância e o maior valor de dominância relativa média (Tab. II). Os componentes da comunidade parasitária de $P$. saltator apresentaram o típico padrão de distribuição superdispersa (Tab. III).

Tabela I. Prevalência, intensidade, intensidade média, abundância média de infecção/infestação e status comunitário dos metazoários parasitos de Pomatomus saltator, do litoral do estado do Rio de Janeiro, Brasil.

\begin{tabular}{|c|c|c|c|c|c|}
\hline Parasitos & $\begin{array}{c}\text { Prevalência } \\
(\%)\end{array}$ & Intensidade & $\begin{array}{l}\text { Intensidade } \\
\text { média }\end{array}$ & $\begin{array}{l}\text { Abundância } \\
\text { média }\end{array}$ & $\begin{array}{c}\text { Status } \\
\text { comunitário * }\end{array}$ \\
\hline \multicolumn{6}{|l|}{ Digenea } \\
\hline Parahemiurus merus (Linton, 1910) & 56,4 & $1-20$ & 6,2 & 3,50 & S \\
\hline Brachyphallus parvus (Menter, 1947) & 72,7 & $1-87$ & 13,1 & 9,50 & C \\
\hline Bucephalopsis callicotyle Kohn, 1962 & 61,8 & $1-49$ & 7,7 & 4,80 & S \\
\hline Didimozóideo imaturo & 3,6 & - & 1,0 & 0,04 & $\mathrm{Sa}$ \\
\hline \multicolumn{6}{|l|}{ Monogenea } \\
\hline Microcotyle pomatomi Goto, 1899 & 87,3 & $1-20$ & 7,0 & 6,10 & C \\
\hline $\begin{array}{l}\text { Gotocotyla acanthophallus (MacCallum \& } \\
\text { MacCallum, 1913) }\end{array}$ & 32,7 & $1-9$ & 2,5 & 0,80 & S \\
\hline \multicolumn{6}{|l|}{ Eucestoda } \\
\hline Scolex sp. & 40,0 & $1-58$ & 12,9 & 5,20 & S \\
\hline Tripanorinquideo não identificado & 52,7 & $1-102$ & 9,6 & 5,10 & S \\
\hline Nybelinia sp. & 1,8 & - & 1,0 & 0,01 & Sa \\
\hline \multicolumn{6}{|l|}{ Acanthocephala } \\
\hline Corynosoma sp. & 52,7 & $1-24$ & 5,4 & 2,80 & $\mathrm{~S}$ \\
\hline \multicolumn{6}{|l|}{ Nematoda } \\
\hline Philometra sp. & 7,3 & $2-3$ & 2,2 & 0,20 & Sa \\
\hline Anisakis sp. & 14,5 & $1-4$ & 1,9 & 0,30 & $\mathrm{Sa}$ \\
\hline Contracaecum sp. & 21,8 & $1-7$ & 2,4 & 0,50 & $\mathrm{Sa}$ \\
\hline Phocanema sp. & 14,5 & $1-8$ & 4,2 & 0,60 & Sa \\
\hline \multicolumn{6}{|l|}{ Copepoda } \\
\hline Caligus pelamydis Kroyer, 1863 & 5,4 & - & 1,0 & 0,05 & Sa \\
\hline Lernanthropus pomatomi Rathbum, 1887 & 32,7 & $1-11$ & 2,6 & 0,80 & $\mathrm{~S}$ \\
\hline
\end{tabular}

*. (C) Espécie central, (S) espécie secundária, (Sa) espécie satélite.

Tabela II. Freqüência de dominância e dominância relativa média dos componentes das infracomunidades de metazoários parasitos de Pomatomus saltator do litoral do estado do Rio de Janeiro, Brasil.

\begin{tabular}{lccc}
\hline \multicolumn{1}{c}{ Parasitos } & $\begin{array}{c}\text { Frequéncia de } \\
\text { dominância }\end{array}$ & $\begin{array}{c}\text { Frequéncia de dominancia } \\
\text { compartilhada }\end{array}$ & $\begin{array}{c}\text { Dominância } \\
\text { relativa média }\end{array}$ \\
\hline Parahemiurus merus & 7 & 2 & $0,097 \pm 0,145$ \\
Brachyphallus parvus & 10 & 1 & $0,175 \pm 0,228$ \\
Bucephalopsis callicotyle & 3 & 0 & $0,079 \pm 0,121$ \\
Microcotyle pomatomi & 19 & 3 & $0,248 \pm 0,254$ \\
Gotocotyla acanthophallus & 3 & 1 & $0,050 \pm 0,139$ \\
Scolex sp. & 5 & 0 & $0,086 \pm 0,168$ \\
Tripanorinquideo năo identificado & 8 & 1 & $0,093 \pm 0,158$ \\
Corynosoma sp. & 2 & 0 & $0,055 \pm 0,084$ \\
Anisakis sp. & 0 & 0 & $0,016 \pm 0,064$ \\
Contracaecum sp. & 0 & 0 & $0,021 \pm 0,073$ \\
Phocanema sp. & 0 & 0 & $0,013 \pm 0,036$ \\
Lernanthropus pomatomi & 2 & 1 & $0,054 \pm 0,152$ \\
\hline
\end{tabular}

Em $P$. saltator, o comprimento total dos espécimes machos $(\mathrm{N}=21)$ e das fêmeas $(\mathrm{N}=34)$, não tiveram diferença significativa $(t=0,435, P=0,665)$. Os espécimes machos apresentaram um comprimento total médio de $41,0 \pm 4,7 \mathrm{~cm}$ e as fềmeas $40,3 \pm 6,4 \mathrm{~cm}$.

Revta bras. Zool. 16 (3): 711 - 723, 1999 
Tabela III. Indice de dispersão (ID) e indice de agregação de Green (IG) dos metazoários parasitos de Pomatomus saltator, do litoral do estado do Rio de Janeiro, Brasil.

\begin{tabular}{lcc}
\hline \multicolumn{1}{c}{ Parasitos } & Indice de dispersão & Indice de Green \\
\hline Parahemiurus merus & 8,029 & 0,036 \\
Brachyphallus parvus & 23,345 & 0,042 \\
Bucephalopsis callicotyle & 20,471 & 0,074 \\
Microcotyle pomatomi & 6,733 & 0,017 \\
Gotocotyla acanthophallus & 3,709 & 0,060 \\
Scolex sp. & 25,817 & 0,087 \\
Tripanorinquideo não identificado & 40,798 & 0,001 \\
Corynosoma sp. & 7,661 & 0,042 \\
Anisakis sp. & 2,098 & 0,078 \\
Contracaecum sp. & 3,361 & 0,084 \\
Phocanema sp. & 5,781 & 0,001 \\
Lernanthropus pomatomi & 4,742 & 0,008 \\
\hline
\end{tabular}

Oito espécies: três digenéticos, um monogenético, um eucestóide e três nematóides mostraram correlação positiva entre o comprimento total do hospedeiro e a abundância parasitária. Duas espécies: um digenético e um nematóide tiveram correlação positiva entre o comprimento total do hospedeiro e a prevalência parasitária. Não houve espécies que mostrassem correlação negativa significativa (Tab. IV).

Brachyphallus parvus $(Z=-2,375, P=0,017 ; F=0,028)$ e Phocanema sp. $(Z=-2,374, P=0,018 ; F=0,018)$, foram as únicas espécies que mostraram influência do sexo sobre as suas abundâncias e prevalências parasitárias. Em B. parvus, os hospedeiros machos apresentaram os valores maiores (16,7 e 90,0\%); e em Phocanema sp., as fềmeas (4,2 e 23,5\%).

Tabela IV. Valores do coeficiente de correlação por postos de Spearman (rs) e do coeficiente de correlação de Pearson ( $r$ ) para avaliar o relacionamento entre o comprimento total de Pomatomus saltator e a abundância e prevalência dos componentes de sua comunidade parasitária ( $P=$ nivel de significância).

\begin{tabular}{lcrrr}
\hline \multicolumn{1}{c}{ Parasitos } & rs & $\mathrm{P}$ & $\mathrm{r}$ & $\mathrm{P}$ \\
\hline Parahemiurus merus & 0,382 & 0,003 & 0,9490 & 0,050 \\
Brachyphallus parvus & 0,586 & $<0,001$ & 0,9150 & 0,085 \\
Bucephalopsis callicotyle & 0,322 & 0,016 & 0,3200 & 0,680 \\
Microcotyle pomatomi & 0,482 & $<0,001$ & 0,5930 & 0,406 \\
Gotocotyla acanthophallus & 0,077 & 0,575 & $-0,1785$ & 0,821 \\
Scolex sp. & 0,085 & 0,534 & 0,2300 & 0,769 \\
Tripanorinquideo năo identificado & 0,303 & 0,024 & 0,7400 & 0,260 \\
Corynosoma sp. & 0,235 & 0,083 & 0,4860 & 0,513 \\
Anisakis sp. & 0,419 & 0,001 & 0,5730 & 0,427 \\
Contracaecum sp. & 0,399 & 0,002 & 0,9830 & 0,017 \\
Phocanema sp. & 0,481 & $<0,001$ & 0,8250 & 0,175 \\
Lernanthropus pomatomi & 0,215 & 0,114 & 0,2540 & 0,746 \\
\hline
\end{tabular}

\section{Infracomunidades parasitárias}

As infracomunidades parasitárias de $P$. saltator tiveram uma diversidade média de $H^{\prime}=1,243 \pm 0,521$ e uma diversidade máxima de 2,011. O índice de uniformidade de Pielou $(J)$ teve uma média de 0,742 $\pm 0,221$. A diversidade mostrou-se correlacionada com o comprimento total do hospedeiro $(r s=0,515, P<0,001)$ 
e não mostrou diferença significativa entre hospedeiros machos $\left(H^{\prime}=1,328 \pm 0,390\right)$ e fềmeas $\left(H^{\prime}=1,191 \pm 0,587\right),(t=0,946, P=0,348)$. A riqueza parasitária teve uma média de 5,6 2,6 (1-12) (Fig. 1).

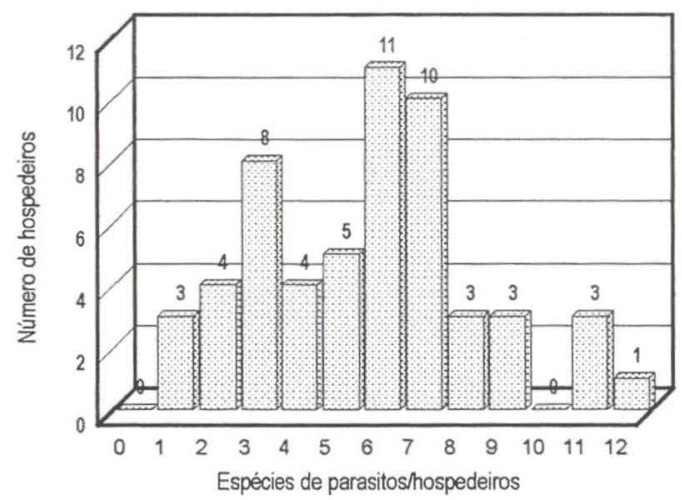

Fig. 1. Distribuição do número de componentes das infracomunidades de metazoários parasitos de Pomatomus saltator do litoral do estado do Rio de Janeiro, Brasil.

As infracomunidades parasitárias foram separadas em três grupos de acordo com a sua biologia, seus locais de infecção/infestação e os recursos utilizados no hospedeiro: ectoparasitos (monogenéticos e copépodes), endoparasitos adultos (digenéticos), e estágios larvares de endoparasitos (larvas de eucestóides, acantocéfalos e nematóides). Os ectoparasitos mostraram associação positiva total $(\mathrm{VR}=1,507)$, e todos os pares de espécies formados apresentaram associação ou covariação significativa (Tab. V). Situação similar apresentou-se nos endoparasitos adultos que mostraram uma associação positiva total (VR = 1,831) (Tab. VI). Nos endoparasitos larvares $(\mathrm{VR}=1,569)$, foram detectados quatro pares de espécies com associação ou covariação positiva e dois com negativa (Tab. VII).

Tabela V. Pares de espécies de ectoparasitos co-ocorrentes em Pomatomus saltator do litoral do estado do Rio de Janeiro, Brasil.

\begin{tabular}{|c|c|c|c|c|}
\hline$x^{2}$ & rs & Microcotyle pomatomi & Gotocotyla acanthophallus & Lemanthropus pomatomi \\
\hline Microcotyle pomatomi & & - & $0,339^{*}$ & $0,391^{*}$ \\
\hline Gotocotyla acanthophallus & & $3,902^{*}$ & - & $0,337^{*}$ \\
\hline Lernanthropus pomatomi & & 1,239 & $6,333^{*}$ & - \\
\hline
\end{tabular}

(rs) Valores do coeficiente de correlação por postos de Spearman; $\left(X^{2}\right)$ valores do teste Qui-quadrado; (*) valores significativos.

\section{DISCUSSÃO}

Os resultados obtidos no presente trabalho, indicam os digenéticos como os principais componentes da comunidade parasitária de $P$. saltator, coincidindo com trabalhos anteriores feitos com peixes marinhos do estado do Rio de Janeiro (LUQUE et al. 1996a; TAKEMOTO et al. 1996; KNOFF et al. 1997). Embora seja importante relacionar este fato com a ecologia e os hábitos alimentares dos hospedeiros 
(HOLMES 1990; LUQUE et al. 1996a), é necessário chamar a atenção de que nos peixes estudados no litoral do Rio de Janeiro, foi encontrado sempre o mesmo padrão (dominância dos digenéticos) de forma independente ao caráter bentônico ou pelágico dos hospedeiros. Embora não se conheçam trabalhos sobre aspectos alimentares das anchovas no litoral sudeste brasileiro, estudos feitos em outras latitudes demonstraram o caráter carnívoro e especializado da dieta de $P$. saltator (SAFINA \& BURGER 1983; SMALE 1986).

Tabela VI. Pares de espécies de endoparasitos adultos co-ocorrentes em Pomatomus saltator do litoral do estado do Rio de Janeiro, Brasil.

\begin{tabular}{|c|c|c|c|c|}
\hline$x^{2}$ & rs & Parahemiurus merus & Brachyphallus parvus & Bucephalopsis callicotyle \\
\hline Parahemiurus merus & & - & 0,186 & $0,280^{*}$ \\
\hline Brachyphallus parvus & & $11,089^{*}$ & - & $0,432^{*}$ \\
\hline Bucephalopsis callicotyle & & $7,326^{*}$ & $10,797^{*}$ & - \\
\hline
\end{tabular}

(rs) Valores do coeficiente de correlação por postos de Spearman; $\left(X^{2}\right)$ valores do teste Qui-quadrado; (*) valores significativos.

Tabela VII. Pares de espécies de larvas de endoparasitos co-ocorrentes em Pomatomus saltator, do litoral do estado do Rio de Janeiro, Brasil.

\begin{tabular}{lcccccc}
\hline$x^{2}$ & rs & Tripanorinquideo & Scolex sp. & Corynosoma sp. & Anisakis sp. Contracaecum sp. Phocanema sp. \\
\hline Tripanorinquideo & - & 0,128 & $0,482^{*}$ & $-0,056$ & 0,248 & $-0,035$ \\
Scolex sp. & 0,596 & - & $0,450^{*}$ & $-0,322^{*}$ & $-0,331^{*}$ & $-0,262$ \\
Corynosoma sp. & $13,173^{*}$ & $8,863^{*}$ & - & $-0,153$ & $-0,138^{*}$ & $-0,049$ \\
Anisakis sp. & 0,359 & $-6,241^{*}$ & $-0,028$ & - & $0,303^{*}$ & $0,698^{*}$ \\
Contracaecum $\mathrm{sp}$. & $5,768^{*}$ & $-6,213^{*}$ & $-0,046$ & $4,359^{*}$ & - & $0,202^{*}$ \\
Phocanema sp. & 0,359 & $-2,950$ & 0,359 & $27,526^{*}$ & 1,350 & - \\
\hline
\end{tabular}

(rs) Valores do coeficiente de correlação por postos de Spearman; $\left(X^{2}\right)$ valores do teste Qui-quadrado; (*) valores significativos.

Algumas variações na composição das infracomunidades parasitárias podem existir em função das peculiaridades ecológicas do litoral brasileiro, o que explicaria a ausência de algumas espécies de endoparasitos registradas em outras latitudes, principalmente no litoral norte-americano (ANDERSON 1970). O alto número de larvas de helmintos coletados de $P$. saltator sugere que mesmo sendo um peixe carnívoro, ocuparia também um nível intermediário na teia trófica marinha, mostrando que poderia ser parte da dieta de elasmobrânquios (hospedeiros definitivos de cestóides tripanorinquídeos e tetrafilídeos) e de mamíferos marinhos (hospedeiros definitivos de Corynosoma sp. e dos anisakídeos).

RÊGO et al. (1983) estudaram a fauna parasitária de $P$. saltator do litoral do Rio de Janeiro. Embora nesse trabalho seja dada maior ênfase ao aspecto taxonômico, os autores acrescentaram também informação quantitativa sobre as espécies de parasitos encontradas. Do ponto de vista qualitativo, RÊGO et al. (1983) coletaram os mesmos táxons parasitários encontrados no presente trabalho, porém, quantitativamente os valores de prevalência (citada por estes autores como incidência de infecção) são bastante menores. Outros aspectos tais como a abundância de infecção/infestação e as associações interespecíficas, foram tratados por RÊGO et al. (1983) baseados apenas em observações feitas no processo de coleta dos parasitos e não medidas ou quantificadas com metodologia estatística adequada. 
O fato de que a maioria dos trabalhos relacionados com ecologia parasitária estejam restritos a períodos não maiores de três anos pode ser considerado um fator limitante para a detecção de padrões na estrutura das comunidades parasitárias (KENNEDY 1993). Comunidades parasitárias de hospedeiros sujeitos à influência de freqüentes alterações ambientais, podem sofrer variações substanciais na sua riqueza e estrutura, tal como foi sugerido por $\operatorname{KENNEDY}(1990,1993)$ para peixes de água doce. Não existem trabalhos deste tipo para peixes marinhos, mas, as diferenças quantitativas obtidas neste trabalho quando comparado com o de RÊGO et al. (1983), podem constituir um primeiro indicativo da necessidade de orientar esforços para a coleta de amostras durante um longo período de tempo com o intuito de avaliar possíveis variações nos níveis de parasitismo (prevalência e abundância parasitária), assim como na estrutura das comunidades parasitárias de peixes marinhos.

Menção especial merecem dois aspectos abordados em RÊGO et al. (1983): a presença de anisakídeos, alertando sobre seu potencial zoonótico e as chamadas "localizações anômalas" dos digenéticos. A respeito, devemos mencionar que mesmo tendo encontrado maiores valores de prevalência das larvas de nematóides, fica difícil estabelecer a possibilidade de uma zoonose, desde que a metodologia usada em ambos trabalhos não incluiu o estudo parasitológico da musculatura somática dos espécimes de $P$. saltator examinados. Quanto às "localizações anômalas", estas são comuns nas necropsias, estando mais relacionadas com o estresse a que o hospedeiro é submetido durante os procedimentos de captura e com o congelamento dos peixes recém capturados, do que com mudanças nos fatores ambientais. Os métodos de captura, não só podem provocar deslocamentos do local de infecção/infestação dos parasitos, mas também influenciam nos aspectos quantitativos das infecções (WILLIAMS et al. 1991).

Outros dois trabalhos fizeram menção a alguns aspectos ecológicos de parasitismo em anchovas, Poulin (1995) citou a presença de seis espécies de parasitos intestinais sem mencionar sua identificação, coletados de anchovas em localidade não especificada. ROHDE et al. (1995), em amostras de anchovas provenientes de Baía de Coffs, Austrália, registraram a presença de cinco espécies de parasitos, sendo a mais prevalente "Microcotylidae sp." com $98,5 \%$ e abundância máxima de 24. Possivelmente, este táxon deve referir-se às duas espécies de microcotilídeos associadas achadas em anchovas no Rio de Janeiro. Ambas, Microcotyle pomatomi e Gotocotyla acanthophallus, são muito parecidas externamente, sendo necessário para diagnóstico, exame detalhado da armadura do átrio genital. O copépode Caligus pelamydis Kroyer, 1863, também foi encontrado por ROHDE et al. (1995) com valores mais altos de prevalência e abundância parasitária. Porém, qualquer comparação feita em relação a estes dois trabalhos deve ser considerada com muita cautela, devido à grande diferença de metodologia e objetivos destes.

Outra característica já observada anteriormente para outros hospedeiros, é a ausência de concentração para dominância de qualquer espécie na comunidade parasitária de $P$. saltator. Isto reforça o mencionado por RoHDE et al. (1995), que usando como modelo os ectoparasitos, postularam que as comunidades parasitárias de peixes marinhos são na realidade complexos pouco ordenados de espécies, fugindo dos padrões interativos comprovados para outros grupos de hospedeiros,

Revta bras. Zool. 16 (3): 711 - 723, 1999 
principalmente aves de ambientes aquáticos. Estes complexos de espécies, próprios das comunidades de parasitos de peixes marinhos neotropicais (LUQUE 1994, 1996; LUQUE et al. 1996a; TAKEMOTO et al. 1996; KNOFF et al. 1997) têm grande importância como modelos para posteriores estudos sobre variações latitudinais e previsibilidade na composição das comunidades parasitárias.

O caráter agregado da distribuição dos parasitos na amostra de anchovas estudada no presente trabalho é considerado um aspecto típico do parasitismo, em função da amplitude das dimensões dos nichos e da heterogeneidade das populações de hospedeiros (ROHDE 1993). LUQUE et al. (1996a) citaram vários exemplos relacionados com parasitos de peixes marinhos. Porém, os mesmos autores mencionaram que algumas espécies de parasitos em função de algumas peculiaridades morfológicas, do seu ciclo biológico e do seu local de infecção, podem apresentar um padrão uniforme de distribuição.

A influência do tamanho do hospedeiro sobre a composição qualitativa e quantitativa das infracomunidades parasitárias, é um tópico bastante documentado. SAAD-FAARES \& COMBES (1992) e LUQUE et al. (1996a), chamaram a atenção para evitar generalizações à respeito. O parasitismo não necessariamente aumenta nos peixes maiores devido a um processo mecânico de acumulação e de maior tempo de exposição às infecções. No caso dos ectoparasitos (de ciclo direto), o relacionamento com o tamanho dos hospedeiros poderá ser influenciado pelo grau de especialização dos órgãos de fixação dos parasitos e pela disponibilidade das formas infectantes a determinadas faixas da população de hospedeiros; e nos endoparasitos (de ciclo indireto, transmitidos troficamente), pelas mudanças dos itens alimentares nas diferentes faixas etárias da população de hospedeiros e pela dinâmica populacional dos hospedeiros intermediários. No caso dos parasitos de $P$. saltator, esta heterogeneidade de padrões na relação tamanho do hospedeiro/prevalência e abundância parasitária, é notória, e sua análise é prejudicada pela falta de trabalhos relacionados com a biologia e aspectos populacionais de $P$. saltator no litoral sudeste brasileiro.

Já a influência do sexo dos hospedeiros, é um aspecto, que mesmo sendo muito citado na literatura, é minimizado nos trabalhos sobre análise comunitária (POULIN 1996). A literatura sobre peixes marinhos, indica que muitos parasitos não apresentam variações quantitativas em relação ao sexo do hospedeiro, e isto é considerado um reflexo da ausência de diferenças na biologia e na dinâmica populacional entre os hospedeiros machos e fêmeas (LUQUE et al. 1996a). Porém, é necessária a realização de experimentos que permitam visualizar a influência de outros fatores que já demonstraram ter maior importância em outros grupos de hospedeiros: aspectos fisiológicos (hormonais e imunológicos), morfológicos e comportamentais dos hospedeiros (BUNDY 1988; LADLE 1992; POULIN 1996). No presente trabalho repete-se o mesmo padrão achado em haemulídeos e carangídeos do Rio de Janeiro (LUQUE et al. 1996a; TAKEMOTO et al. 1996), onde a maioria das espécies não mostraram diferenças na prevalência e abundância parasitária com relação ao sexo dos hospedeiros.

Estudos sobre a diversidade parasitária em peixes marinhos são escassos e foram feitos considerando apenas uma parte das infracomunidades (ecto ou endo- 
parasitos). Trabalhos incluindo a totalidade dos componentes das infracomunidades de peixes marinhos são recentes (GEORGE-NASCIMENTO \& IRIARTE 1989; HoLMES 1990; CAMPOS \& CARBONELl 1994; LUQUE et al. 1996b; TAKEMOTO et al. 1996; KNOFF et al. 1997). Holmes (1990), JANOvY et al. (1992) e POULIN (1995) estabeleceram que fatores históricos e zoogeográficos, entendidos como interações entre a história evolutiva e as características ecológicas dos hospedeiros, determinam a diversidade das suas comunidades parasitárias. Poulin (1995) concluiu que em peixes, a riqueza de endoparasitos é proporcional com o aumento de alimento animal na dieta dos hospedeiros, entretanto, o mesmo autor mencionou que a riqueza de ectoparasitos não mostra associação com as variáveis ecológicas (tamanho do corpo, dieta, habitat, latitude). Porém, os resultados obtidos em recentes trabalhos feitos no Rio de Janeiro, e no presente trabalho, demonstraram uma escassez de padrões definidos para a diversidade parasitária, observando-se principalmente um relacionamento direto com o tamanho dos hospedeiros, padrão considerado comum para peixes de água doce por BELL \& BURT (1991). Isto, deverá ser analisado em função das características ecológicas do litoral brasileiro, região de encontro de correntes marítimas frias e quentes, com áreas de ressurgência e afloramento (VALENTIN 1994), que podem influenciar na ecologia das populações de hospedeiros intermediários e definitivos dos parasitos.

Nas últimas duas décadas, vários trabalhos foram publicados sobre o caráter das comunidades parasitárias. STOCK \& HoLMES (1988) consideraram os dois tipos de comunidades parasitárias: interativa (com espécies centrais e evidências de relacionamentos interespecíficos) e a isolacionista (com ausência de espécies centrais e de relacionamentos interespecíficos), não como uma dicotomia na qual seriam classificadas todos os tipos de comunidades parasitárias, mas, como as extremidades de um continuum formado por um conjunto de comunidades com características intermediárias entre o tipo interativa e isolacionista. A comunidade parasitária de P. saltator, apresentou várias espécies com alta prevalência e abundância parasitária e um número importante de pares de espécies associadas e correlacionadas significativamente, mostrando maior proximidade do tipo interativo, além de ser diferente das comunidades parasitárias de outros peixes pelágicos, como por exemplo os do gênero Oligoplites, estudados por TAKEMOTO et al. (1996), no Rio de Janeiro, que mostraram comunidades tipicamente isolacionistas. Isto demonstra a necessidade de se continuar acumulando informações sobre as comunidades parasitárias de peixes marinhos desta região, para conferir e comparar padrões estruturais em hospedeiros com distintas características ecológicas, comportamentais e populacionais.

\section{REFERÊNCIAS BIBLIOGRÁFICAS}

AMATo, J.F.R. 1983a. Digenetic Trematodes of Percoid fishes of Florianópolis, southern Brasil - Homalometridae, Lepocreadiidae and Opecoelidae, with the description of seven new species. Rev. Brasil. Biol. 43: 73-98.

1983b. Digenetic Trematodes of Percoid fishes of Florianópolis, southern Brasil - Pleorchiidae, Didymozoidae and Hemiuridae, with the description of three new species. Rev. Brasil. Biol. 43: 99-124. 
Anderson, H.G. 1970. Annotated list of parasites of the bluefish Pomatomus saltatrix. Tech. Pap. Bur. Sport Fish. Wild. 54: 1-15.

BELL, G. \& A. BURT. 1991. The comparative biology of parasite species diversity: internal helminths of freshwater fish. Jour. Anim. Ecol. 60: 1047-1064.

BunDY, D.A.P. 1988. Sexual effects on parasite infection. Parasitol. Today 4: 186-189.

BusH, A.O. \& J.C. Holmes. 1986. Intestinal helminths of lesser scaup ducks: an interactive community. Can. Jour. Zool. 64: 142-152.

BusH, A.O.; J.M. AHO \& C.R. KENNEDY. 1990. Ecological versus phylogenetic determinants of helminth parasite community richness. Evol. Ecol. 4: 1-20.

Bush, J.O.; K.D. Lafferty; J.M. Lotz \& A.W. ShostaK. 1997. Parasitology meets ecology on its own terms: Margolis et al. revisited. Jour. Parasitol. 83: 575-583.

Campos, A. \& E. Carbonell. 1994. Parasite community diversity in two Mediterranean labrid fishes Symphodus tinca and Labrus merula. Jour. Fish Biol. 44: 409-413.

Esch, G.W.; A.W. Shostak; D.J. Marcogliese \& T.M. Goater. 1990. Patterns and processes in helminth parasite communities, p.1-19. In: G. EsCH; A.O. BUSH \& J. Aно (Eds). Parasite communities: patterns and processes. New York, Chapman \& Hall, 335p.

Figueiredo, J.L. \& N. Menezes. 1980. Manual de Peixes Marinhos do Sudeste de Brasil III. Teleostei (2). São Paulo, Museu de Zoologia, Universidade de São Paulo, 90p.

George-NAscimento, M. \& J.L. Iriarte. 1989. Las infracomunidades de parásitos metazoos del chancharro Helicolenus lengerichi Norman, 1937 (Pisces, Scorpaenidae): un ensamble no interactivo de especies. Rev. Chil. Hist. Nat. 62: 217-227.

GOMES, D.C.; S.P. FÁBIO \& F.J.T. RoLAS. 1972. Contribuição para o conhecimento dos parasitos de peixes do litoral do Estado da Guanabara - Parte I. Mem. Inst. Oswaldo Cruz 70: 541-553.

Holmes, J.C. 1990. Helminth communities in marine fishes, p.101-130. In: G. EsCH; A.O. Bush \& J. AHO (Eds). Parasite communities: patterns and processes. New York, Chapman \& Hall, 335p.

Janovy, J.; R.E. Clopton \& T.J. Percival. 1992. The roles of ecological and evolutionary influences in providing structure to parasite species assemblages. Jour. Parasitol 78: 630-640.

KENNEDY, C.R. 1990. Helminth communities in freshwater fish: structured communities or stochastic assemblages, p.131-153. In: G. EsCH, A.O. BusH \& J. Aно (Eds). Parasite communities: patterns and processes. New York, Chapman \& Hall, 335p.

1993. The dynamics of intestinal helminth communities in eels Anguilla anguilla in a small stream: long-term changes in richness and structure. Parasitology 107: 71-78.

KNoFf, M.; J.L. LuQUE \& J.F.R. AMATO. 1997. Community ecology of the metazoan parasites of grey mullets, Mugil platanus (Osteichthyes: Mugilidae) from the littoral of the State of Rio de Janeiro. Rev. Brasil. Biol. 57: 441-454. 
KoHN, A. \& P.F. BÜHRNHEIM. 1971. Ocorrência de Microcotyle pomatomi Goto, 1899 (Polistomata, Microcotylidae) na Baía de Guanabara. Atas Soc. Biol. Rio de Janeiro 14: 131-133.

KoHN, A.; D.C. GoMes \& U. BÜHRnheIM. 1971. Gotocotyla travassosi sp. n., gastrocotilídeo de brânquias de enchova (Polistomata). Mem. Inst. Oswaldo Cruz 69: 49-51.

KoHN, A.; C.P. SANTOS \& M.F.D. BAPTISTA-FARIAS. 1992. New host records and localities of some monogenea from Brazilian marine fishes with scanning electron microscopy of Bicotylophora trachinoti (Mac Callum, 1921). Mem. Inst. Oswaldo Cruz 87 (Suppl. 1): 109-114.

LadLE, R.J. 1992. Parasites and sex: catching the Red Queen. Trends Ecol. Evol. 7: 405-408.

LUDWIG, J.A. \& J.F. REYNOLDS. 1988. Statistical Ecology: A primer on methods and computing. New York, Wiley-Interscience Publications, 337p.

LUQUE, J.L. 1994. Dinámica poblacional y estructura de la comunidad de metazoarios parásitos de Menticirrhus ophicephalus (Pisces: Sciaenidae) en la costa peruana. Rev. Biol. Trop. 42: 21-29.

-1996. Distribución y asociaciones interespecíficas en las comunidades de metazoarios ectoparásitos de peces esciénidos del Perú. Rev. Biol. Trop. 44: 387-394.

LuQUE, J.L. \& M.E. Oliva. 1993. Análisis cuantitativo y estructura de la comunidad parasitaria de Paralonchurus peruanus (Pisces: Sciaenidae) en la costa peruana. Parasitol. al Día 17: 107-111.

LuQUE, J.L.; J.F.R. AMATO; \& R.M. TAKEMOTO. 1996a. Comparative analysis of the communities of metazoan parasites of Orthopristis ruber and Haemulon steindachneri (Osteichthyes: Haemulidae) from the southeastern Brazilian littoral: I. structure and influence of the size and sex of hosts. Rev. Brasil. Biol. 56: 279-292.

. 1996b. Comparative analysis of the metazoan parasite communities of Orthopristis ruber and Haemulon steindachneri (Osteichthyes: Haemulidae) from the southeastern Brazilian littoral: II. diversity, interspecific associations, and distribution of the gastrointestinal parasites. Rev. Brasil. Biol. 56: 293-302.

POULIN, R. 1995. Phylogeny, ecology, and the richness of parasite communities in vertebrates. Ecol. Monog. 65: 283-302.

-1996. Sexual inequalities in helminth infections: a cost of being a male. Amer. Nat. 147: 287-295.

RÊGo, A.A., J.J. Vicente, C.P. Santos \& R.M. Wekid. 1983. Parasitas de anchovas, Pomatomus saltatrix (L.) do Rio de Janeiro. Ciên. Cult. 35: 1329-1336.

RoHDE, K. 1993. Ecology of marine parasites. An introduction to marine parasitology. Wallingford, Oxon, CAB International, 297p.

RoHDE, K.; C. HAYWARD \& M. HEAP. 1995. Aspects of the ecology of metazoan ectoparasites of marine fishes. Int. Jour. Parasitol. 25: 945-970.

SAAD-FARES, A. \& C. COMBES. 1992. Abundance/host size relationships in a fish trematode community. Jour. Helminthol. 66: 187-192.

SAFINA, C. \& J. BURGER. 1983. Interactions among free-living bluefish and prey 
fish in an ocean environment. Oecologia 79: 91-95.

SCHLUTER, D. 1984. A variance test for detecting species associations, with some example association. Ecology 65: 998-1005.

SMALE, M.J. 1986. The feeding habits of six pelagic and predatory teleosts in eastern Cape coastal waters (South Africa). Jour. Zool. London 201: 357-409.

STOCK, T.M. \& J.C. HoLmES. 1988. Functional relationships and microhabitat distributions of enteric helminths of grebes (Podicipedidae): the evidence for interactive communities. Jour. Parasitol. 74: 214-227.

STONE, J.E. \& D.B. PENCE. 1978. Ecology of helminth parasitism in the bobcat from West Texas. Jour. Parasitol. 64: 295-302.

TAKEMOTO, R.M.; J.F.R. AMATO \& J.L. LUQUE. 1996. Comparative analysis of the metazoan parasite communities of leatherjackets, Oligoplites palometa, $O$. saurus and $O$. saliens (Osteichthyes: Carangidae) from Sepetiba Bay, Rio de Janeiro, Brazil. Rev. Brasil. Biol. 56: 639-650.

Travassos, L.; J.F.T. Freitas \& A. Kohn. 1969. Trematódeos do Brasil. Mem. Inst. Oswaldo Cruz 67: 1-886.

Valentin, J.L. 1994. A ressurgência. Fonte de vida dos oceanos. Ciência Hoje 18: 19-25.

Wallet, M. \& A. KoHN. 1987. Trematodes parasites de Poissons marins du littoral de Rio de Janeiro, Brésil. Mem. Inst. Oswaldo Cruz 82: 21-27.

Williams, E.H.; L. BunKLEY-Williams; M.J. Dowgiallo \& W.G. DyeR. 1991. Influence of collection methods on the occurrence of alimentary canal helminth parasites in fish. Jour. Parasitol. 77: 1019-1022.

ZAR, J.H. 1996. Biostatistical Analysis. New Jersey, Prentice-Hall, Inc., $3^{\text {rd }}$ ed., $662 \mathrm{p}$.

Recebido em 13.I.1998; aceito em 12.VII.1999. 